\title{
EVIDÊNCIAS CIENTIFICAS SOBRE O USO DA ESPECTROSCOPIA RAMAN CONFOCAL IN VIVO NA PELE HUMANA
}

\author{
Aline Priscila Campos Pereira ${ }^{1}$ \\ Michely Glenda Pereira da Silva ${ }^{2}$
}

Resumo: A Espectroscopia Raman Confocal (ERC) é uma técnica totalmente não invasiva, eficaz na caracterização em tempo real dos arranjos químicos dos tecidos biológicos vivos. Com isso, o objetivo desse trabalho é destacar as pesquisas com uso da ERC. Foram selecionados e analisados das bases de dados: PubMed e Web of Science: 18 artigos científicos. Foram apresentados em dois quadros, obedecendo a ordem: nome dos autores, ano, revista, número de participantes, região espectral, tipo de sistema Raman Confocal, tipo e potência dos lasers. Todos os artigos reportados neste trabalham ressaltam que a ERC trata se de uma ferramenta valiosa, a qual fornece dados confiáveis. Conclui-se que existem poucos estudos científicos utilizando a ERC na pele humana, principalmente in vivo, apesar de fornecer informações em diferentes profundidades e obter dados com uma metodologia totalmente invasiva.

Palavras-chave: Água Corporal; Análise espectral Raman; Pele.

\footnotetext{
1 Graduação em Ciências Biológicas pela Universidade do Vale do Paraíba, Brasil. Iniciação Científica no Laboratório de Espectroscopia Raman Confocal/Instituto de Pesquisa e Desenvolvimento, Brasil. E-mail: allinneferrara@gmail.com

2 Enfermeira, Mestre em Engenharia Biomédica pela Universidade do Vale do Paraíba - UNIVAP. Doutoranda em Engenharia Biomédica - Bolsista da Coordenação de Aperfeiçoamento de Pessoal de Nível Superior CAPES/Instituto de Pesquisa e Desenvolvimento - IP\&D/Universidade do Vale do Paraíba - UNIVAP, Brasil. Email: michely.glenda@hotmail.com.
} 